\title{
The Regulatory Network of Cyclic GMP-AMP Synthase-Stimulator of Interferon Genes Pathway in Viral Evasion
}

\section{OPEN ACCESS}

Edited by:

Chunfu Zheng,

University of Calgary, Canada

Reviewed by:

Longwei Zhao,

Wenzhou Medical University, China

E. Angela Murphy,

University of South Carolina,

United States

*Correspondence:

Chen Wang

cwang1971@cpu.edu.cn

Ye Cui

Ye.cui2@childrens.harvard.edu

Quanyi Wang

quanyiwang@cpu.edu.cn

${ }^{t}$ These authors have contributed

equally to this work

Specialty section:

This article was submitted to

Virology,

a section of the journal

Frontiers in Microbiology

Received: 07 October 2021

Accepted: 04 November 2021

Published: 13 December 2021

Citation:

Hu TY, Pan MY, Yin Y, Wang C,

Cui $Y$ and Wang $Q$ (2021) The

Regulatory Network of Cyclic

GMP-AMP Synthase-Stimulator

of Interferon Genes Pathway in Viral Evasion. Front. Microbiol. 12:790714.

doi: 10.3389/fmicb.2021.790714
Tongyu Hu' ${ }^{1 \dagger}$, Mingyu Pan ${ }^{1 \dagger}$, Yue Yin ${ }^{1}$, Chen Wang ${ }^{1 *}$, Ye Cui ${ }^{2,3 *}$ and Quanyi Wang ${ }^{1 *}$

1 State Key Laboratory of Natural Medicines, Department of Life Science and Technology, China Pharmaceutical University, Nanjing, China, ${ }^{2}$ Division of Immunology, The Boston Children's Hospital, Boston, MA, United States, ${ }^{3}$ Department of Pediatrics, Harvard Medical School, Boston, MA, United States

Virus infection has been consistently threatening public health. The cyclic GMP-AMP synthase (cGAS)-Stimulator of Interferon Genes (STING) pathway is a critical defender to sense various pathogens and trigger innate immunity of mammalian cells. cGAS recognizes the pathogenic DNA in the cytosol and then synthesizes $2^{\prime} 3^{\prime}$-cyclic GMPAMP ( $\left(2^{\prime} 3^{\prime}\right.$ cGAMP). As the second messenger, cGAMP activates STING and induces the following cascade to produce type I interferon (IFN-I) to protect against infections. However, viruses have evolved numerous strategies to hinder the cGAS-STING signal transduction, promoting their immune evasion. Here we outline the current status of the viral evasion mechanism underlying the regulation of the cGAS-STING pathway, focusing on how post-transcriptional modifications, viral proteins, and non-coding RNAs involve innate immunity during viral infection, attempting to inspire new targets discovery and uncover potential clinical antiviral treatments.

Keywords: viral evasion, cGAS-STING, type I interferon, innate immune, post-translational modification

\section{INTRODUCTION}

Infectious diseases are the top threats to public health. With the persistent invading of various pathogens, mammalians have developed certain strategies to protect themselves from extraneous risks. During infection, germline-encoded pattern recognition receptors (PRRs) recognize the pathogen and damage-associated molecular patterns (PAMPs and DAMPs), such as the viral nucleic acids (DNA or RNA), which initiate subsequent immune responses, and orchestrate an elaborate defense system against infection (Carty et al., 2021; Li and Chang, 2021).

The cGAS-STING pathway is one of the predominant and conserved mechanisms of the host to eliminate pathogens through several aspects, including IFN-I, releasing, autophagy initiation, proinflammatory cytokines production, and cell death pathways (Phelan et al., 2020; Fang and Peng, 2021; Zhao et al., 2021). Viruses have optimized their evading tactics for superior replication and spreading to counteract host immunity. For instance, due to their genetic flexibility, viruses have 
developed various viral proteins and non-coding RNAs to interrupt several checkpoints of cGAS-STING. Besides, they craftily confuse the host regulatory system to diminish immune responses and engage viral escaping from immunity (Li et al., 2019; Bouayad, 2020; Kikkert, 2020).

The models of most immune evasion during innate immune responses are conserved, including altering the post-transcriptional modifications (PTMs) of vital proteins to inactivate or degrade these components, eluding DNA sensing from PRRs, decreasing cyclic GMP-AMP (cGAMP) cellular abundance, and modifying metabolism approach in host cells (Eaglesham and Kranzusch, 2020). Intriguingly, novel mechanisms are continuously uncovered, which elucidate a more concrete picture of cGAS-STING involvement in viral evasion. Nonetheless, how pathogens avoid detection and clearance by immune systems needs to be more comprehensively elucidated. Herein, we have summarized newly emerging hot spots of cGAS-STING regulation in viral evasion and summarized frontier advances in relevance processes. The present review provides potential worth evaluating targets in innate immune response that are viable in clinical trials and antiviral reagents development for current and future studies.

\section{THE CASCADE OF CYCLIC GMP-AMP SYNTHASE-STIMULATOR OF INTERFERON GENES PATHWAY DURING VIRAL INVASION}

At the first stage of viral invasion, virus-derived doublestranded DNAs (dsDNAs) trigger the conformational change and activation of cGAS. Activated cGAS catalyzes and releases the second messenger $2^{\prime}, 3^{\prime}$-cGAMP, which binds to STING, an adaptor located at endoplasmic-reticulum (ER)-membrane (Chen et al., 2016). This process induces oligomerization of STING and its traveling from ER to the Golgi via ER-Golgi intermediate compartment (ERGIC) (Hopfner and Hornung, 2020). Translocation and structure switch of STING provides the prerequisite for TANK-binding kinase 1 (TBK1) recruitment and auto-phosphorylation. TBK1 induces phosphorylation of STING C-terminal tail (CTTs) motif, which supplies a docking site for interferon regulatory factor 3 (IRF3) (Shang et al., 2019; Zhao et al., 2019). Moreover, recent studies suggest that recruitment of TBK1 to STING may perform a more significant role in antagonist virus infection and restrict oncogenesis (Figure 1), which expands the horizon of cGAS-STING axis function besides IRF3 and nuclear factor- $\mathrm{B}(\mathrm{NF}-\kappa \mathrm{B})$ signaling (Yum et al., 2021). Accordingly, IRF3 is phosphorylated by TBK1 and subsequently dimerized, resulting in IRF3 nuclear translocation and transcriptional activation, further inducing IFN-I release (Zhang et al., 2020c). Besides, STING activation also contributes to the recruitment of IКB kinase (IKK) and facilitates NF$\kappa \mathrm{B}$ inhibitor I $\kappa \mathrm{B} \alpha$ phosphorylation. Activated NF- $\kappa \mathrm{B}$, similar to activated IRF3 dimer, translocates into the nucleus and generates downstream pro-inflammatory cytokines formation (Motwani et al., 2019).
As a signaling cascade of pathogen sensing and (IFNI) production, IFN- $\beta$ binds to IFN $\alpha / \beta$ receptor 1 (IFNAR 1 ) and IFNAR2, which launch interferon-stimulated genes (ISGs) production through Janus kinase signal transducer and activator of transcription (JAK-STAT) pathway (Wan et al., 2020). Activated JAK1 and tyrosine kinase 2 (TYK2) trigger signal transducers and activators of transcription 1 (STAT1)/STAT2 phosphorylation. IRF9 is then recruited to STAT1/2 heterodimers to constitute the IFN-I-stimulated gene factor 3 (ISGF3) complex and initiate ISGs production (Schneider et al., 2014). In conclusion, the autocrine and paracrine of IFN-I- $\beta$ consist of dynamic feedback to regulate the cGAS-STING axis (Wang et al., 2020a).

Additionally, cGAS-STING signaling participates in IRF3independent viral-sensing and triggers autophagy, an ancestral antiviral process of host defense (Yamashiro et al., 2019; Hopfner and Hornung, 2020). The interaction of STING and LC3 leads to non-canonical autophagy initiation without classic autophagy regulators, such as Unc-51-like kinase 1 (ULK1) protein kinase complex (Birgisdottir et al., 2013; Liu et al., 2019). Golgi-oriented STING vesicle trafficking provides a conjugation and lipidation membrane platform for LC3, enabling autophagosome formation (Gui et al., 2019). Besides, STING activation also results in V-ATPase-ATG16L1induced LC3B lipidation on single-membrane perinuclear vesicles, presumably to cripple invaded viruses by facilitating lysosomal fusion (Fischer et al., 2020). Furthermore, the recent advance of crosstalk between STING and autophagy indicates that STING translocating to the Golgi is indispensable to switch on DNA- and RNA-virus triggered autophagy (Zhang $\mathrm{R}$. et al., 2021). In addition, cGAS is demonstrated to interact with the autophagy protein Beclin-1 as well. This event suppresses the cGAMP synthesis and releases the RUBICON, an autophagy inhibitor, which increases autophagic clearance of viral DNA to prevent the overactivation of cGAS-STING signaling (Liang et al., 2014).

Accumulating studies show that the cGAS-STING pathway is widely implicated in the DNA-sensing process, including viral clearance and autoimmune diseases (Lin and Zheng, 2019; Brezgin et al., 2021; Zheng, 2021). It is important to broaden our comprehension of each step of the cGASSTING signaling to finetune the immune responses. Moreover, utilizing this pathogen defense pathway supplies valuable guidance to predict potential antiviral therapeutics and drug developments, which hinders viral evasion from host innate immune surveillance.

\section{POST-TRANSLATIONAL MODIFICATION OF CYCLIC GMP-AMP SYNTHASE-STIMULATOR OF INTERFERON GENES PATHWAY COMPONENTS IS SIGNIFICANT TO AVOID VIRAL EVASION}

Several studies showed that viruses benefit from hijacking host enzymes to alter the modification of antiviral immune 


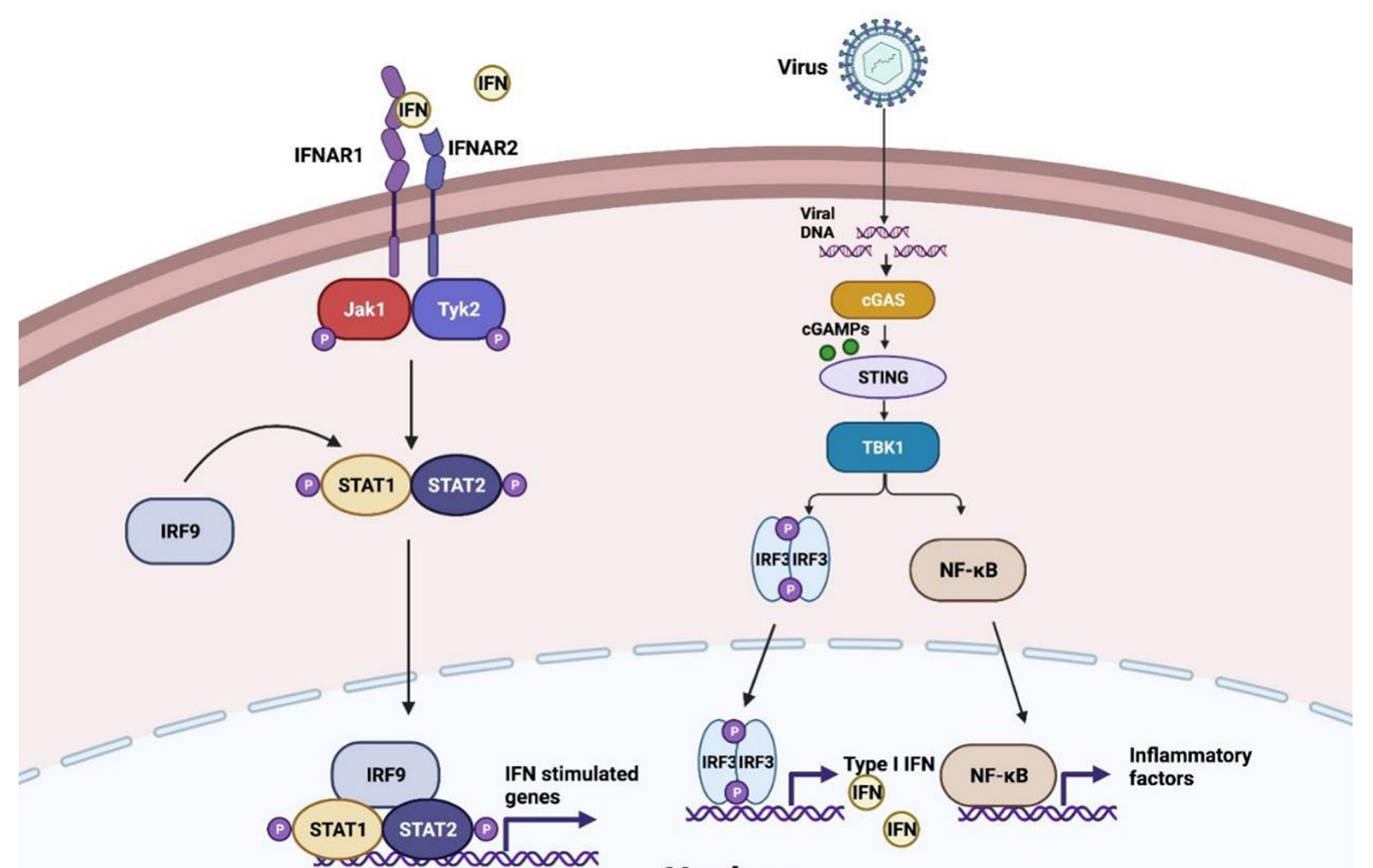

Nucleus

FIGURE 1 | cGAS-STING pathway in virus invasion. After virus infection, cyclic GMP-AMP synthase (cGAS) senses the viral DNA to synthesize cyclic GMP-AMPs (cGMPs) and activate stimulator of interferon genes (STING) to recruit TANK-binding kinase 1 (TBK1) and IкB kinase (IKK), and then interferon regulatory factor 3 (IRF3) and nuclear factor- $\mathrm{KB}(\mathrm{NF}-\mathrm{\kappa} B$ ) are induced to translocate into the nucleus and generate the production of IFN-I- $\beta$ and inflammatory factors. IFN-I- $\beta$ conducts Janus kinase signal transducer and activator of transcription (JAK-STAT) pathway activation and initiates interferon-stimulated genes transcription.

cascades component proteins, thus conducive to their replication (Carty et al., 2021; Hong et al., 2021; Song et al., 2021). Understanding the details of PTMs in cGAS-STING signaling helps interrupt virus evasion. Here we summarize the current findings of PTM regulating on different components in the cGASSTING pathway.

\section{Post-transcriptional Modifications of Cyclic GMP-AMP Synthase Ensure Functional Protection From Viral Infection}

When the viral DNAs are released in the cytoplasm, cGAS efficiently recognizes and processes the enthetic DNA ligands to synthesize cGAMP. Virus infection has been found to induce various forms of post-translational modifications at different sites of cGAS, which manipulates the synthetase capability of cGAS and its affinity to pathogenic DNA (Table 1; Wu and Li, 2020; Bhowmik and Zhu, 2021; Song et al., 2021).

To maintain the homeostasis of cGAS, several PTMs play an important role in down-regulating cGAS function to prevent its excessive activation. Upon virus infection, cGAS is phosphorylated by DNA-dependent protein kinase (DNA-PK), hindering its oligomerization and enzymatic activity (Sun et al., 2020). The glutamylation of cGAS is also identified after DNA virus infections. The dynamic regulation of cGAS glutamylation, either mono-, or poly-, impedes its synthase activity and DNA binding capability, which adjusts the strength of immune response to pathogens (Xia et al., 2016). The enzymes involved in cGAS glutamylation are concluded in Table 1. Additionally, recent research revealed cGAS was symmetrically dimethylated at Arg124 residue by protein arginine methyltransferase 5 (PRMT5). As a result, cGAS could not bind the DNA ligands, and the antiviral response was dampened during HSV-1 infection (Ma et al., 2021).

Proper activation of cGAS is important to generate IFN-I production and subsequent antiviral immunity. Sentrin/SUMOspecific protease 7 (SENP7) rescues cGAS inhibition by removing the small ubiquitin-like modifier (SUMO) from Lys335, 372, and 382 of cGAS (Cui et al., 2017). The activity of cGAS can also be dynamically regulated by acetylation. Acetylation of cGAS at Lys384, Lys394, and Lys414, which are close to its $\mathrm{C}$ terminal, keeps cGAS at a quiescent state. Sensing abnormal DNA ligands triggers histone deacetylase 3 (HDAC3) to deprive the acetyl groups of cGAS to switch on its enzymatic activity (Dai et al., 2019). In contrast, lysine acetyltransferase 
TABLE 1 | Enzymes involved in cGAS PTMs.

\begin{tabular}{|c|c|c|c|c|c|}
\hline Target protein & PTM sites (h:human, m:mice) & PTM & Enzymes & Function & References \\
\hline \multirow[t]{10}{*}{ cGAS } & T68, S213(h) & Phosphorylation & DNA-PK & Hinders cGAS oligomerization & Sun et al., 2020 \\
\hline & $\mathrm{E} 272(\mathrm{~m})$ & Polyglutamylation & TTLL6 & Impedes cGAS DNA-binding ability & Xia et al., 2016 \\
\hline & E302(m) & Mono-glutamylation & TTLL4 & Suppresses cGAS synthase activity & Xia et al., 2016 \\
\hline & $\mathrm{E} 272(\mathrm{~m})$ & Depolyglutamylation & CCP6 & Activates cGAS & Xia et al., 2016 \\
\hline & $\mathrm{E} 302(\mathrm{~m})$ & Demonoglutamylation & CCP5 & Activates cGAS & Xia et al., 2016 \\
\hline & K335/372/382(m) & De-SUMOylation & SENP7 & Reverses cGAS inhibition & Cui et al., 2017 \\
\hline & $\mathrm{R} 124(\mathrm{~m})$ & Methylation & PRMT5 & Interrupts cGAS binding with DNA & Ma et al., 2021 \\
\hline & K384/394/414(h) & Deacetylation & HDAC3 & Maintains cGAS response to DNA & Dai et al., 2019 \\
\hline & $\mathrm{K} 47 / 52 / 62 / 83(\mathrm{~m})$ & Acetylation & KAT5 & Increases DNA-binding of cGAS & Song et al., 2020 \\
\hline & K231/421(h) & Polyneddylation & RNF111 & Improves cGAS dimerization & Li et al., 2021b \\
\hline
\end{tabular}

5 (KAT5) mediating the acetylation on the $\mathrm{N}$ terminal of cGAS at Lys47/52/62/83 promotes the activation of cGAS (Song et al., 2020). Contemporary research has exhibited the poly-neddylation of cGAS with the presence of the Ube2mRnf111 axis. The neddylation of cGAS conserved residues K231 and K421 are crucial to facilitate cGAS dimerization and promote its cytoplasmic DNA binding ability. As a result, these modifications restrict HSV-1 infection in vivo (Li et al., 2021b).

Acting as the initial DNA-sensor to induce robust innate immune responses, the enzymatic activity and stability of cGAS are precisely regulated. To protect the host from the threat of virus, more PTM forms and sites of cGAS need further investigation.

\section{The Modification of Stimulator of Interferon Genes by Ubiquitin Maintains Stimulator of Interferon Genes Relevant Antiviral Responses}

STING, the adaptor downstream of cGAS, is also the center molecule of most DNA-sensing pathways. Multiple PTMs have been found to dominate STING intracellular trafficking, conformational change, and activation during infection (Li et al., 2020; Hong et al., 2021). One of the most common modifications that dynamically regulates STING activity is ubiquitination. Different STING ubiquitin linkage types accomplish diverse functions to STING relevance immune cascades (Davis and Gack, 2015). In this section, we focus on the ubiquitination of STING during viral infection (Table 2).

STING can be ubiquitinated with K48-linked polyubiquitination, which leads to its proteasome degradation. The immune system has evolved this strategy as a negative feedback loop to balance normal immune responses and autoimmunity. TRIM29, an E3 ligase, can be induced by DNA virus stimulation, which catalyzes K48-linked ubiquitination of STING on Lys288/337 and mediates STING degradation (Li et al., 2018). Similarly, K48-linked ubiquitination of STING at Lys150 by RNF90 also negatively regulates the DNA-sensing pathway (Yang et al., 2020). The ubiquitination mediated degradation process can be reversed by deubiquitinases, which maintain STING function. The deubiquitinases OTUD5 and ubiquitin-specific protease (USP) 44 deprive the K48-linked polyubiquitin chains of STING at Lys347 and Lys236, respectively (Zhang et al., 2020a).

Distinct from K48 ubiquitination mediated degradation, K63linked ubiquitination promotes the activation of the substrates. STING can also be modified by K63-linked ubiquitination at Lys20/150/224/236 by tripartite motif protein 32 (TRIM32), which of these are essential for STING activation and interaction with TBK1 (Zhang et al., 2012). On the contrary, USP49 antagonizes STING activation by removing its K63-linked ubiquitin chains, impedes STING aggregation, and subsequent TBK1 recruitment after HSV-1 invasion (Ye et al., 2019). Similarly, infected by DNA virus, the Myb-like, SWIRM, and MPN domains 1 protein (MYSM1) is increasingly expressed and interacts with STING, leading to the removal of K63linked ubiquitination STING at Lys150 to down-regulate STING signaling (Tian et al., 2020). Intriguingly, a recent observation reveals that a novel autophagy receptor, CCDC50, can recognize K63-polyubiquitinated STING for autophagic degradation, which inhibits IFN-I and pro-inflammatory cytokines production. Moreover, CCDC50 deficiency restricts HSV-1 replication, which shows a possible therapeutic strategy to prevent viral evasion (Hou et al., 2021).

STING is standing at the crossroad of IFN-I releasing, non-classical autophagy initiation, and NF- $\kappa$ B activation. The ubiquitin-related regulation is quite crucial for the stability and function of STING to prevent viral immune evasion.

\section{Appropriate Modification of TANK-Binding Kinase 1 Defends Viral Invasion}

TBK1, the downstream component of STING in the cGASSTING axis, its kinase activity is indispensable to IFN-I generation and virus-induced autophagy initiation (Sparrer et al., 2017). Multi-categories of post-translational modifications of TBK1 involve modulating the strength of cGAS-STING signaling activation (Table 3 ).

Ubiquitination and acetylation are also engaged in TBK1 regulation. Virus sensing induced the expression of E3 ubiquitin ligase RNF128. K63-linked ubiquitin chains are continuously added to TBK1 sites at Lys30 and Lys401, triggering TBK1 activation and following IFN-I- $\beta$ release (Song et al., 2016). 
TABLE 2 | Dynamic ubiquitin decorations of STING.

\begin{tabular}{|c|c|c|c|c|c|}
\hline Target protein & PTM sites & Enzyme & Decoration type & Function & References \\
\hline \multirow[t]{7}{*}{ STING } & K288/337 & TRIM29 & Ubiquitination (K48) & Promotes STING proteasomal degradation & Li et al., 2018 \\
\hline & $\mathrm{K} 150$ & RNF90 & Ubiquitination (K48) & Induces STING degradation & Yang et al., 2020 \\
\hline & K236 & USP44 & Deubiquitination (K48) & Promotes STING stability & Zhang et al., 2020a \\
\hline & K347 & OTUD5 & Deubiquitination (K48) & Prevents STING degradation & Guo Y. et al., 2021 \\
\hline & K20/150/224/236 & TRIM32 & Ubiquitination (K63) & Increases STING interaction with TBK1 & Zhang et al., 2012 \\
\hline & - & USP49 & Deubiquitination (K63) & Improves STING aggregation and translocation & Ye et al., 2019 \\
\hline & K150 & MYSM1 & Deubiquitination (K63) & Attenuates STING activation & Tian et al., 2020 \\
\hline
\end{tabular}

TABLE 3 | Modifications of TBK1 in regulating IFN-I production.

\begin{tabular}{|c|c|c|c|c|c|}
\hline Target protein & PTM sites & PTM & Enzymes & Function & References \\
\hline \multirow[t]{8}{*}{ TBK1 } & $\mathrm{K} 30 / 401$ & Ubiquitination (K63) & RNF128 & Activates TBK1 & Song et al., 2016 \\
\hline & K670 & Deubiquitination (K33) & USP38 & Induces subsequence ubiquitination & Lin et al., 2016 \\
\hline & K670 & Ubiquitination (K48) & DTX4 and TRIP & Promotes TBK1 degradation & Lin et al., 2016 \\
\hline & $\mathrm{K} 30 / 401$ & Deubiquitination (K63) & USP15 & Represses TBK1 activation & Huang et al., 2020 \\
\hline & K344 & Ubiquitination (K27) & NEDD4 & Induces selective autophagy of TBK1 & Xie et al., 2021 \\
\hline & K241/692 & Deacetylation & HDAC3 & Enables TBK1 kinase activity & Tang et al., 2021 \\
\hline & C637 & S-glutathionylation & GSTM1 & Inhibits TBK1 phosphorylation & Wang et al., 2020b \\
\hline & W354/394 & Tyrosine-phosphorylation & Lck/Hck/Fgr & Impedes TBK1 activation & Liu et al., 2017 \\
\hline
\end{tabular}

USP38 exclusively removes K33-linked poly-ubiquitination of TBK1 at Lys670, which is consecutively replaced by K48-linked ubiquitin chains attributed by DTX4 and TRIP, causing the proteasomal degradation of TBK1 (Lin et al., 2016). Additionally, with the assistant of UBE2S, USP15 is recruited to TBK1 and removes K63-linked polyubiquitin chains of TBK1. This process represses IFN-I- $\beta$ production and provides an advantageous element for virus proliferation (Huang et al., 2020). Another reported E3 ubiquitin ligase, ASB8, is a negative regulator of IFN-I signal transduction. Mechanistically, after viral infection, ASB8 interacts with TBK1/IKKi kinase complex and promotes the K48-linked ubiquitination of TBK1/IKKi, which is degraded by proteasome afterward (Guo et al., 2020). Furthermore, NEDD4 drives the K27-linked poly-ubiquitination of TBK1 at Lys344 to instigate selective autophagy clearance of TBK1 (Xie et al., 2021). HDAC3 also involves deacetylation of TBK1 at Lys241 and Lys692, enabling TBK1 kinase activity (Dai et al., 2019). In turn, TBK1 mediates HDAC3 phosphorylation to enhance the deacetylase activity of HDAC3, which generates a feedback mechanism. The deficiency of HDAC3 impairs IFN-I releasing, therefore promoting viral replication in mice (Tang et al., 2021).

Several enzymes engage in distinct TBK1 modifications to prevent TBK1 from excessive activation upon viral stimulation. Previous studies reveal that the Src family kinases (SFKs) Lck, Hck, and Fgr restrict IFN-I production. During virus infection, Lck/Hck/Fgr can directly phosphorylate TBK1 at Tyr354/394 to restrain TBK1 dimerization and activation as a feedback approach in antiviral immunity (Liu et al., 2017). Recently, Wang et al. (2020b) investigated that a highly conserved cysteine residue C637 of TBK1 could be S-glutathionylated by glutathione S-transferase M1 (GSTM1). This special modification of TBK1 inhibits its phosphorylation at Ser172, hence regulating the release of IFN-I in the process of virus infection (Wang et al., 2020b).

\section{The Non-canonical Post-translational Changes of Interferon Regulatory Factor 3 Affect Immune Evasion of Virus}

Last but not the least component regulated by PTMs in the cGASSTING pathway is IRF3. The transcription factor IRF3 plays a commander-like role in manipulating IFN- $\beta$ transcription upon viral infection. After translocated into the nucleus, IRF3 interacts with CREB-binding protein (CBP)/p300 to initiate downstream genes transcription (Schwanke et al., 2020). During pathogens infection, the host facilitates PTMs to alter the conformation and activity of IRF3, which accordingly performs antagonism between viruses and the host immune signaling (Table 4).

Besides phosphorylation and classical ubiquitination, increasingly novel PTMs are verified of their involvements in regulating IRF3 intention. Atypical ubiquitination in cGASSTING signal cascades is still barely reported. Zhang et al. (2020e) identified the K6-linked ubiquitination of IRF3 at Lys39/98/105 under viral infection, which is essential for its DNA binding ability. Moreover, Zhang et al. (2020e) team uncovered that the ovarian tumor domain-containing 1 (OTUD1) can deubiquitinate K6-, K11-, and K29-linked ubiquitination of IRF3. The ubiquitin-like protein ISG15 conjugates with target proteins and induces ISGylation, proven crucial during viral invasion and evasion (Dzimianski et al., 2019; Chiang et al., 2021; Mathieu et al., 2021). The ISG15 E3 ligase HERC5 adds ISG15 to IRF3 at Lys193/360/366 to counteract with Pin1 induced IRF3 polyubiquitination, guaranteeing the stability of an IRF3 structure (Shi et al., 2010). Nuclear receptor-binding SET domain 3 (NSD3) directs the Lys366 monomethylation of IRF3, shielding 
TABLE 4 | Modifications of IRF3 during virus infection.

\begin{tabular}{|c|c|c|c|c|c|}
\hline Target protein & PTM sites (h:human,m:mice) & PTM & Enzymes & Function & References \\
\hline \multirow[t]{5}{*}{ IRF3 } & K98 (h) & Deubiquitination (K6) & OTUD1 & Restricts IRF3 DNA binding & Zhang et al., 2020e \\
\hline & $\mathrm{K} 193 / 360 / 366(\mathrm{~m})$ & ISGylation & HERC5 & Stabilizes IRF3 & Shi et al., 2010 \\
\hline & K366(m) & Monomethylation & NSD3 & Ensures IRF3 subsequent phosphorylation & Wang et al., 2017 \\
\hline & K366(m) & Dephosphorylation & PP1cc & Dephosphorylate IRF3 & Wang et al., 2017 \\
\hline & K359(m) & Acetylation & KAT8 & Reduces IRF3 induced gene transcriptions & Huai et al., 2019 \\
\hline
\end{tabular}

the phosphatase PP1Cc-mediate IRF3 dephosphorylation, thus intensifying the transcriptional regulator function of IRF3 and following IFN-I release (Wang et al., 2017). IRF3 is also acetylated at Lys359 by lysine acetyltransferase 8 (KAT8) to attenuate virus-induced IFN-I generation. IRF3 acetylation interrupts its association with interferon genes promoters, hence invalidating over-committed immune response in vivo (Huai et al., 2019).

Collectively, accumulating evidence indicates that the post-translational modifications involved in the cGAS-STING pathway are quite significant. The improvement of protein structure analysis techniques has broadened the approaches for researchers to validate additional decorations of target proteins. A growing number of new PTMs and the relative functional aspects can be recognized. Delineating the complicated network of PTMs control of cGAS-STING can contribute to the present state of antagonizing viral evasion strategies investigations.

\section{MANIPULATION OF CYCLIC GMP-AMP SYNTHASE-STIMULATOR OF INTERFERON GENES AXIS BY VIRAL PROTEINS}

Struggling with the defense mechanism of host immunity, viruses themselves have developed several means to create a more convenient environment for replication (Zhang et al., 2016; Lange et al., 2021; Sausen et al., 2021). For instance, viruses utilize their proteins to control the host's innate-immune signaling pathways for evasion (Table 5). This section concentrates on the direct interaction between viral proteins and the pivotal component in the cGAS-STING pathway (Figure 2). Addressing critical interplay between virus and host interferon responses will contribute to further therapeutic procedure researches.

\section{Viral Deubiquitinases in Regulating Cyclic GMP-AMP Synthase-Stimulator of Interferon Genes Pathway}

The dynamic modification of host proteins maintains the homeostasis in innate immune signaling regulation. Multiple viruses encode viral deubiquitinase (DUB) to interrupt the antiviral responses in host cells, aiming to attenuate innate immune system activation, such as the cGAS-STING axis (Kumari and Kumar, 2018; Proulx et al., 2021).

pUL48, a DUB encoded by human cytomegalovirus (HCMV), removes K63 ubiquitination of STING to attenuate IFN-I induction and promote carcinogenesis (Kumari et al., 2017).
Transmissible gastroenteritis virus (TGEV) also utilizes its papain-like protease (PL1) to antagonize IFN- $\beta$ expression through deubiquitination of STING (Hu et al., 2017). Analogously, the herpes simplex virus (HSV) DUB VP1-2 immediately interacts with Lys150-ubiquitinated STING and removes its K63-linked ubiquitination, inducing viral evasion in the brain (Bodda et al., 2020). In addition, the HSV-1 ubiquitinspecific protease (UL36USP) dampens I $\mathrm{B} \alpha \alpha$ degradation via its deubiquitinase activity, further restricting NF- $\kappa \mathrm{B}$ signaling activation to dysregulate host immune response. Likewise, UL36USP also decreases the IFN- $\beta$ cascade response upon HSV-1 infection (Ye et al., 2017).

The severe acute respiratory syndrome coronavirus (Sars$\mathrm{CoV}$ ) membrane-anchored PLpro domain (PLpro-TM) is elucidated its function of disrupting STING-TRAF3-TBK1 complex formation, decreasing the ubiquitination level of the complex components, ultimately impairing IRF3 phosphorylation and dimerization (Chen et al., 2014). The papain-like protease (PLpro) domain of SCoV cleaves the viral polyprotein and promotes viral spreading (Klemm et al., 2020). Although sharing high sequence identity with SCoV-PLpro, PLpro of SCoV2 prefers to remove the ubiquitin-like protein ISG15 modification of the host protein rather than ubiquitin, reflecting a different tendency for substrate selection. Upon invasion, SCoV2-PLpro was implicated in IRF3 de-ISGylation to inhibit IFN-I production (Shin et al., 2020).

\section{The Viral Tegument Proteins in Suppressing Innate Immune Responses}

The tegument is a protein cluster that fills the space between the nucleocapsid and the envelope expressed in all herpes viruses. The tegument proteins are essential for the viral envelope and viral DNA containing capsid transport (Yang et al., 2019). Apart from virus enveloping, different kinds of tegument proteins have evolved diverse strategies to suppress host immunity.

Lately, the tegument proteins of HSV-1 have been reported to be involved in disrupting cGAS-STING induced IFN-I production. For instance, UL37 deamidates a crucial asparagine site of cGAS to eliminate cGAMP composition, deactivating IFNI initiation cascades after infection (Zhang et al., 2018). Another tegument protein, UL46 encoded by HSV-1, obstructs TBK1 dimerization, facilitating declining IFN-I production and leading to HSV-1 immune evasion (You et al., 2019). Moreover, during HSV-1 infection, the tegument protein UL41 acts as the RNase to degrade cGAS mRNA, which contributes to HSV-1 evading of DNA-sensing pathway (Su and Zheng, 2017). Phase separation is a result of forming large biomolecules clusters and lately 
TABLE 5 | Viral proteins involved in cGAS-STING cascade signaling.

\begin{tabular}{|c|c|c|c|c|c|c|}
\hline Genre & Genome & Virus & Viral protein & $\begin{array}{l}\text { Target } \\
\text { proteins }\end{array}$ & Function & References \\
\hline \multirow[t]{6}{*}{ Deubiquitinase (DUB) } & DNA & HCMV & pUL48 & STING & Deubiquitination & Kumari et al., 2017 \\
\hline & & HSV-1 & VP1-2 & STING & & Bodda et al., 2020 \\
\hline & & & UL36USP & $\mid \kappa B \alpha$ & & Ye et al., 2017 \\
\hline & RNA & TGEV & PL1 & STING & & Hu et al., 2017 \\
\hline & & SARS-CoV & PLpro & $\begin{array}{l}\text { STING-TRAF3- } \\
\text { TBK1 } \\
\text { complex }\end{array}$ & & Chen et al., 2014 \\
\hline & & SARS-CoV2 & PLpro & $\mathrm{IRF3}$ & De-ISGylation & Shin et al., 2020 \\
\hline \multirow[t]{10}{*}{ Tegument protein } & DNA & VZV & ORF9 & cGAS & Restricts cGAS-DNA condensates & Xu G. et al., 2021 \\
\hline & & HSV-1 & UL37 & cGAS & Impairs cGAMP synthesis & Zhang et al., 2018 \\
\hline & & & UL46 & TBK1 & Inhibits TBK1 dimerization & You et al., 2019 \\
\hline & & & UL41 & cGAS & Degrades cGAS mRNA & Su and Zheng, 2017 \\
\hline & & & VP22 & cGAS & Restrains cGAS catalyze activity & Huang et al., 2018 \\
\hline & & & ORF52NP22 & cGAS & Inhibits cGAS-DNA phase separation & Xu G. et al., 2021 \\
\hline & & HCMV & UL23 & STAT1 & Impedes STAT1 phosphorylation & Feng et al., 2021 \\
\hline & & & UL94 & STING & Inhibits STING dimerization & Zou et al., 2020 \\
\hline & & & pp65 & cGAS & Hampers cGAMP synthesis & Biolatti et al., 2018 \\
\hline & & GPCMV & GP83 & cGAS & Inhibits cGAS activity & Choi et al., 2021 \\
\hline \multirow[t]{5}{*}{ Accessory protein } & DNA & HSV-1 & Us11 & Hsp90 & Restricts Hsp90-TBK1 complex formation & Liu et al., 2018 \\
\hline & RNA & SCoV2 & ORF3a & STING & Obstructs STING triggered NF-кB activation & Rui et al., 2021 \\
\hline & & & ORF9b & TBK1 & Decreases TBK1 phosphorylation & Han et al., 2021 \\
\hline & & HIV-2, SIV & Vpx & STING & Diminishes STNG function in NF-кB initiation & Su et al., 2019 \\
\hline & & HIV-1 & Vpr & Karyopherins & Dampens IRF3 and NF-kB nuclear translocation & Khan et al., 2020 \\
\hline
\end{tabular}

has explicated its role in intracellular immune signal regulation (Xiao et al., 2021). VP22 of HSV-1 has previously determined its function in impeding the cGAMP synthetase activity of cGAS (Huang et al., 2018). Xu G. et al. (2021) further clarified that gamma- and alpha-herpes tegument proteins ORF52, VP22, and ORF9 effectively disrupt the cGAS-DNA condensation as cGAS-DNA phase separation inhibitors to countermeasure viral clearance in host cells.

The HCMV tegument protein UL23 interacts with STAT1 and hinders STAT1 phosphorylation from optimizing subsequent viral dissemination (Feng et al., 2021). UL94 of HCMV represses translocation and dimerization of STING to facilitate virus replication; UL82 and UL35 also antagonize cGAS-STING signaling separately at STING trafficking and TBK1 level (Fu et al., 2017; Fabits et al., 2020; Zou et al., 2020). Moreover, the HCMV pp65 (pUL83) selectively associates with cGAS and disrupts its following signal transduction with STING, supporting HCMV evading from innate immunity (Biolatti et al., 2018). In other species, Choi et al. (2021) uncovered the role of guinea pig cytomegalovirus (GPCMV) tegument protein GP83, the supposed HCMV pp65 homolog, in epithelial cell infection. GP83 interacts with the DNA sensors IFI16 and cGAS, particularly in targeting cGAS, which shares a conserved function with pp65 (Choi et al., 2021).

\section{Accessory Proteins of Viruses Against Host Antiviral Immunity}

Accessory proteins have different purposes and quantities in many viruses and focus more on viral spreading, evasion, and host immune regulation rather than manipulating viral replication (Fang P. et al., 2021).

Upon infection, herpes simplex virus 1 (HSV-1) accessory protein Us11 prevents Hsp90 interaction with TBK1, disrupting Hsp90-TBK1 complex formation. Moreover, Us11 also induces TBK1 proteasomal degradation. These processes of Us11 facilitate HSV-1 replication by reducing IFN-I- $\beta$ generation (Liu et al., 2018). Vpx, a virion-associated accessory protein encoded by human immunodeficiency virus-2 (HIV-2) and certain simian immunodeficiency virus (SIV), binds STING to facilitate NF- $\kappa$ B complex organization and inhibit NF- $\kappa B$ signal transduction (Su et al., 2019). Further investigation reveals that HIV-1 accessory protein Vpr manipulates innate immunity to promote HIV-1 replication, showing another virus's evasion strategy. Mechanically, Vpr prevents IRF3 and NF$\kappa \mathrm{B}$ import to nuclear by interacting with karyopherin, hence antagonizing downstream antiviral responses (Khan et al., 2020). SARS-CoV-2 accessory protein ORF3a interacts with STING to diminish intranuclear p65 accumulation, impeding NF$\kappa \mathrm{B}$ signaling initiation without affecting IRF3 triggered IFN-I generation (Rui et al., 2021). Han et al. (2021) reported that SARS-CoV-2 ORF9b interacted with TBK1 and dysregulated TBK1 phosphorylation to evoke escaping SARS-CoV-2.

Diverse viral proteins are a virus-involved strategy against host immunity. The persistent appearance of novel variants of viruses causes health emergencies to humanity. Mastering the function of viral proteins and how they manipulate the innate immune system helps us prevent viral evasion and ultimately reduce risks from virus-induced infection. 


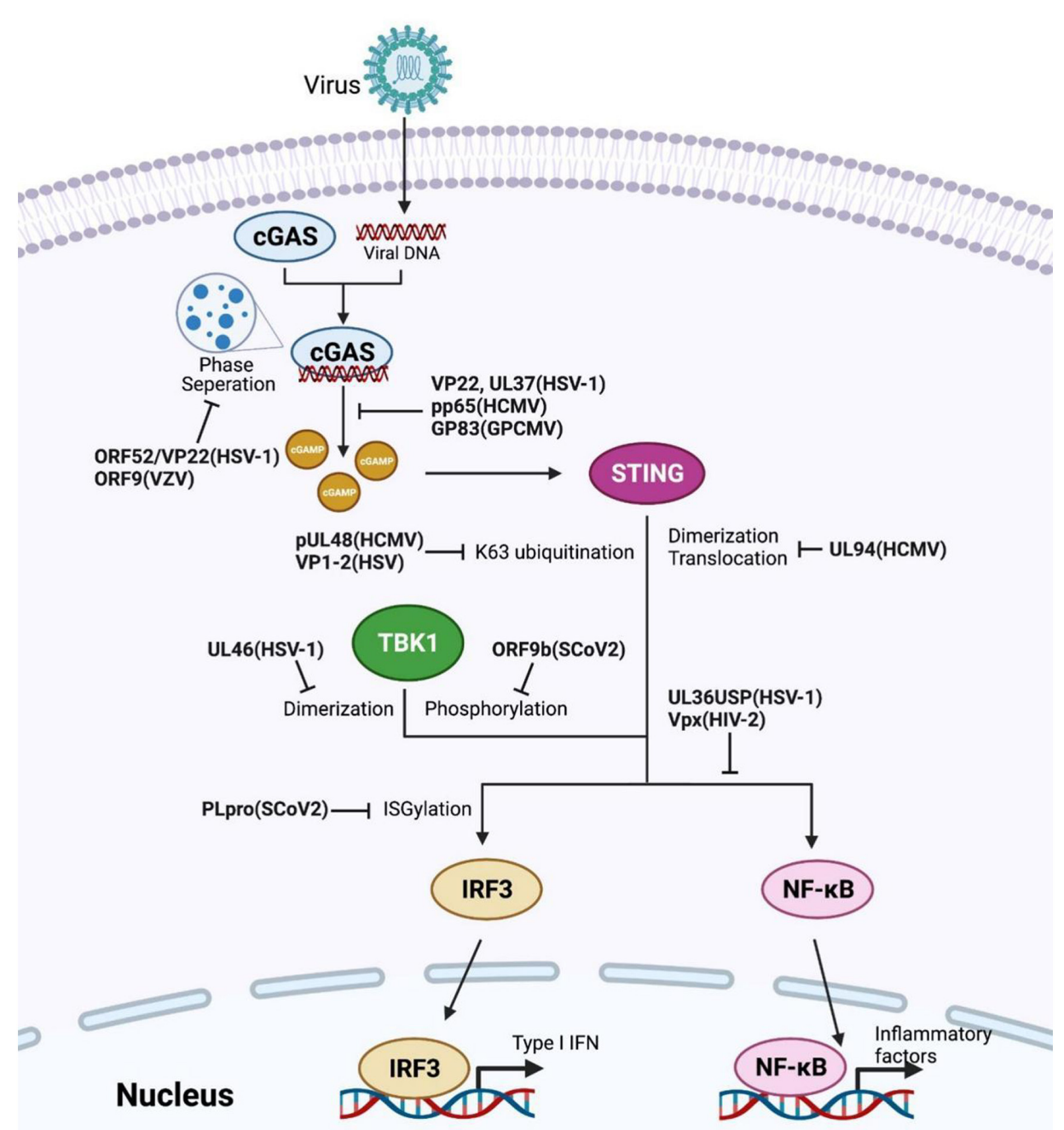

FIGURE 2 | Viral proteins induced evasion strategies by restricting the cGAS-STING axis. Several reported viral proteins dysregulate different checkpoints in the cGAS-STING pathway, such as invalidating the enzyme activity of cGAS or TBK1, obstructing STING activation, altering the modifications of key components. The corresponding viral proteins and their species are labeled in bold.

\section{REGULATION OF NON-CODING RNA IN VIRAL EVASION}

RNA-centric management of host-virus interactions is increasingly causing attention (Gokhale et al., 2021). This section has a brief systematical review of the function and mechanism of non-coding RNA implicated in virus-induced immune defense underlying the cGAS-STING pathway (Table 6).

Host immunity engages diverse RNA-directive strategies to operate vital proteins expression in IFN-I signaling. For instance, long non-coding RNAs (lncRNAs) contain more than 200 nucleotides and regulate gene expression at transcriptional or post-transcriptional levels (Kesheh et al., 2021). After being infected by Kaposi's sarcoma-associated herpes virus (KSHV), the lncRNA NEAT1 and HEXIM1 from a special ribonucleoprotein complex interact with
cGAS is required to initiate foreign DNA triggered cGASSTING activation. The viral protein ORF52 can disrupt the interplay of HEXIM1-cGAS and induce KSHV evasion of immune response (Morchikh et al., 2017). Using functional screening of host lncRNAs, Wang et al. (2020b) revealed IncRNA-GM, an enhancer of TBK1 activity by interacting with glutathione S-transferase M1 (GSTM1), turns to reduce TBK1 S-glutathionylation. Virus invasion represses the abundance of lncRNA-GM in host macrophages, facilitating immune escaping under viral infection (Wang et al., 2020b). Moreover, $\mathrm{Qu}$ et al. (2021) reported that the circular RNA (circRNA) AIVR, an innovative lncRNA expressed in A549 cells, absorbed the microRNA (miRNA) bound to the positive regulatory protein of IFN-I- $\beta$ generation, CREBBP. Deficient in AIVR expression dampens antiviral reaction in host cells (Qu et al., 2021). 
TABLE 6 | Regulations of non-coding RNA in virus invasion.

\begin{tabular}{|c|c|c|c|c|}
\hline Type & Species & ncRNA & Function & References \\
\hline IncRNA & Human and mouse & IncRNA-GM & Represses TBK1 S-glutathionylation & Wang et al., 2020b \\
\hline circRNA & Human & AIVR & Increases CREBBP expression & Qu et al., 2021 \\
\hline \multirow[t]{6}{*}{ miRNA } & Teleost fish & $\mathrm{miR}-15 b$ & Represses TBK1 expression & Chang et al., 2020 \\
\hline & Teleost fish & $\mathrm{miR}-210$ & Represses STING expression & Xu et al., 2018 \\
\hline & Human & miR-576-3p & Decreases STING expression & Geddes et al., 2018 \\
\hline & Cat & miR-101, miR-26a & Downregulate SOCS5 & Zhang et al., 2020b \\
\hline & HCMV & miR-US33as-5p & Disrupts IFN-IAR1 function & Zhang Q. et al., 2021 \\
\hline & HSV-1 & $\mathrm{miR}-\mathrm{H} 2-3 \mathrm{p}$ & Suppresses DNA sensing by DDX41 & Duan et al., 2019 \\
\hline
\end{tabular}

The miRNAs are members of small non-coding RNAs. Multiple DNA and RNA viruses are capable of encoding miRNA to accelerate their propagation or promote immune evasion in the host (Nanbo et al., 2021). Current studies suggest that Siniperca chuatsi rhabdovirus (SCRV) can utilize the host miR-15b and miR-210 of teleost fish, whose expressions are pronouncedly enhanced during viral infection. SCRV participates separately in TBK1 and STING expression to manipulate IFNI responses, promoting its replication and immune escape (Xu et al., 2018; Chang et al., 2020). In another research, Geddes et al. (2018) filter and determine the function of miR-576-3p in human hepatocarcinoma cell line $\mathrm{HuH}-7$. With the expansion of the Oropouche virus, miR-576-3p decreases the expression of STING to restrict IFN- $\beta$ related immune responses, which facilitates pathogenesis in the organism (Geddes et al., 2018). Feline herpesvirus 1 (FHV-1) also induces upregulation of cats miR-101 and miR-26a to stifle viral trespass in a cGASdependent way. These miRNAs target and repress the IFN-I-I negative regulator cytokine signaling 5 (SOCS5) to potentiate host immune response while preventing viral evasion (Zhang et al., 2019, 2020b). HCMV encoded miR-US33as-5p can bind IFNAR1 and dysregulate following ISGs expression. Accordingly, resistance to IFN-I induced viral elimination (Zhang Q. et al., 2021). Host DNA sensor Asp-Glu-Ala-Asp (DEAD)-box helicase 41 (DDX41) is capable of mediating STING-induced IRF3 activation (Briard et al., 2020). Besides, a recent study has identified that miR-H2-3p of HSV-1 downregulates the mRNA and protein level of DDX41 to affect IFN- $\beta$ production, which promotes HSV-1 replication at the same time (Duan et al., 2019).

RNA regulatory mechanisms that participate in virus defense immunity are potential candidates for therapeutic targeting. Numerous non-coding RNAs remain unknown. Therefore, future studies need to pay more attention to these special RNAs.

\section{SEVERE ACUTE RESPIRATORY SYNDROME CORONAVIRUS-2 INFECTION AND CYCLIC GMP-AMP SYNTHASE-STIMULATOR OF INTERFERON GENES}

There are still numerous coronaviruses that have been uncovered and need to be analyzed (Hilgenfeld, 2014). In recent decades, the world has suffered from multiple coronaviruses, from SARS to the Middle East respiratory syndrome (MERS), and now, COVID-19. The world is expecting to find out effective antiviral therapeutics against this global pandemic. cGAS-STING is a pivotal antiviral pathway that has been recently proved by several studies of its involvement in SARS-CoV-2 infection (Berthelot and Liote, 2020; Berthelot et al., 2020; Liu et al., 2021). Here, we conclude many ongoing studies that focus on the cGAS-STING pathway as a therapeutic target to block the evasion of SARS-CoV-2.

Separate groups of researchers have reached an agreement that direct activation of STING can robustly block SARS-CoV-2 infection. Moreover, they found that the STING agonist, diABZI and diABZI-4, can effectively restrict SARS-CoV-2 replication (Chipurupalli et al., 2020; Zhu et al., 2021). Furthermore, Wu et al. (2021) expanded the function of a novel STING agonist, $\mathrm{CDG}^{S F}$, as an adjuvant for the SARS-CoV-2 vaccine. Compared with other coronaviral proteins, PLpro contributes to both virus replication and host cell signaling-cascade regulation, which is more suitable to be a target for antiviral drug design (Baez-Santos et al., 2015). Using protease activitybased and high-throughput screening methods, two valuable SCoV2-PLpro inhibitors, tanshinone IIA sulfonate sodium, and chloroxine, are selected and show their potential in clinical treatment for COVID-19 (Xu Y. et al., 2021). Ma et al. (2021) identify Jun9-72-2 and Jun9-75-4 as the representatives of several SCoV2-PLpro inhibitors, with higher affinity than previously reported inhibitor GRL0617. Another study has determined that combined administration of cGAMP and viruslike particles (VLPs) vaccine perform a worth noting effect in strengthening vaccine immunogenicity (Chauveau et al., 2021). These promising antiviral drug candidates shore up the tough struggle with the virus and encourage researchers to be more concerned about viral evasion mechanisms.

\section{CONCLUSION AND FUTURE PERSPECTIVES}

The cGAS-STING pathway is evolutionarily conserved in mammalian species and has intriguing functions in other species (Cheng et al., 2020; Morehouse et al., 2020). Recent studies have revealed diverse regulation of the cGAS-STING pathway during virus infection and subsequent innate immune evasion of different viruses in distinct host species (Zheng, 2018; 
Zhu and Zheng, 2020; Guo Y. K. et al., 2021; Yu H. et al., 2021; Yu P. et al., 2021). Despite the conserved strategies we have concluded above, the emergence of more and more novel schemes shows the diversity and complicated network manipulation of cGAS-STING signal cascades during viral escaping, presenting a cat-rat race of survival between viruses and their hosts.

Viral immune escape factors craftily control cGAS-STING signal transduction from beginning to end. Intriguingly, at the first step of infection, human papillomaviruses (HPVs) have evolved a special vesicular trafficking method, which can translocate viral genome (vDNA) into host intranuclear environment without being detected by surveillance of abnormal DNA ligands, therefore bypassing the cGAS-STING pathway (Uhlorn et al., 2020). Then, the second messenger, cGAMP, is produced after detecting viral DNA and acts as the immunostimulator of the cGAS-STING pathway. Poxvirus immune nucleases (poxins) from mammalian and insecticidal poxvirus have been recently defined for their participation in cGAMP degradation. They have also abolished downstream STING signal cascades reaction (Eaglesham et al., 2019, 2020). Several processes also take part in regulating key component functions of the cGAS-STING pathway. Marek's disease virus (MDV) major oncoprotein Meq hampers the combination of IFN regulatory factor 7 (IRF7) and TBK1 with STING, which facilitates MDV-induced lymphomagenesis in avians ( $\mathrm{Li}$ et al., 2019). Another finding that was previously reported is that capsid protein of MDV, VP23, also participates in the cGAS-STING blockade by impeding TBK1 phosphorylation of IRF7 (Gao et al., 2019). The DP96R gene of the African swine fever virus (ASFV) suppresses TBK1 phosphorylation and inhibits IKK $\beta$, contributing to the evasion of ASFV from immune clearance (Wang et al., 2018). A recent study uncovers that the interaction of STING with sulfated glycosaminoglycans (sGAGs) is essential to exercise the STING function. Decreased expression of Slc35b2 hampers the sulfate process of GAGs. Thus, the STING polymerization is impeded, blunting the immune responses to vaccinia virus infection (Fang R. et al., 2021). Presenting these evasion tactics of the virus provides insight into targeting novel antiviral countermeasures.

Apart from adjusting signal delivery by key proteins, several strategies regulate the intensity of antiviral response at the mRNA level. Li et al. (2021a) suggest that the RNA-binding protein LUC7L2 participates in the negative feedback of virus-induced immunity by interaction with STING precursor mRNA, which represses STING expression. IFN-I signaling activation also induces proteasome degradation of WT1associated protein (WTAP) to decrease m6A modifications of IRF3 and IFNAR1 mRNAs, which negatively regulates antiviral responses (Ge et al., 2021). Moreover, recent findings also reveal that people of STING haplotype are more sensitive to dengue virus (DENV) protease than homozygote genotype, whose STING is risky to be cleaved during viral infection. This research discusses how the diverse STING genetic background affects DENV pathogenesis and provides another orientation for future precision medicine development (Su et al., 2020).

Viruses are the mainspring of infectious diseases, several carcinogenic processes, and have caused immeasurable public health for years. Understanding how viruses adjust the innate immune system affords probabilities to cure virus-related diseases and prevent viral infection. However, simply knowing the principles and patterns is merely a beginning. Antiviral drug development is an urgent issue to humanity to diminish the impact of virus disturbance and prevent the evasion of viruses. Acting as the key checkpoints in maintaining the interferon homeostasis in vivo, several valuable targets in cGAS-STING signal transduction offer inspiration for antiviral drugs invention.

Recently, multiple anticancer drugs have been repurposed of their capabilities in antiviral treatments (Aldea et al., 2021; Xu Y. et al., 2021). For instance, $\beta$-arrestin 2 is a regulator of $G$ protein-coupled receptor (GPCR) signaling pathways, promoting cGAMP production to regulate the cGASSTING axis by targeting cGAS positively. During the viral invasion, $\beta$-arrestin 2 is degraded by the ubiquitin-proteasome system, which causes the decrease of IFN- $\beta$ level in host cells and viruses evasion. Apart from its known efficacy in curing heart disease, Carvedilol is re-screened as the blocker of virus-induced $\beta$-arrestin 2 degradations to rescue the diminished antiviral immune response, which provides a novel candidate for antiviral drug research and development (Zhang et al., 2020d).

Notwithstanding all these mechanisms about virus escape we have discussed above, there are still innumerable details that need further exploration. The appearance of drug-resistant variants and novel viruses with high pathogenicity are bound to immeasurable economic and public health damages. There still is a long way to go with this tug of war between humans and viruses.

\section{AUTHOR CONTRIBUTIONS}

TH drafted the manuscript, and prepared figures with BioRender.com. YY, MP, QW, and YC revised the manuscript. CW edited and reviewed the final version of this manuscript. All authors contributed to the article and approved the submitted version.

\section{FUNDING}

The present study was supported by the National Natural Science Foundation of China (31730018 and 81672029), the Open Project of State Key Laboratory of Natural Medicines (SKLNMZZCX201802), the "Double First-Class" Project of China Pharmaceutical University (CPU2018GF10), and the Jiangsu Innovative and Entrepreneurial Talents Program. 


\section{REFERENCES}

Aldea, M., Michot, J. M., Danlos, F. X., Ribas, A., and Soria, J. C. (2021). Repurposing of anticancer drugs expands possibilities for antiviral and antiinflammatory discovery in COVID-19. Cancer Discov. 11, 1336-1344. doi: 10.1158/2159-8290.CD-21-0144

Baez-Santos, Y. M., St John, S. E., and Mesecar, A. D. (2015). The SARScoronavirus papain-like protease: structure, function and inhibition by designed antiviral compounds. Antiviral Res. 115, 21-38. doi: 10.1016/j. antiviral.2014.12.015

Berthelot, J. M., and Liote, F. (2020). COVID-19 as a STING disorder with delayed over-secretion of interferon-beta. EBioMedicine 56:102801. doi: 10 . 1016/j.ebiom.2020.102801

Berthelot, J. M., Liote, F., Maugars, Y., and Sibilia, J. (2020). Lymphocyte changes in severe COVID-19: delayed over-activation of STING? Front. Immunol. 11:607069. doi: 10.3389/fimmu.2020.607069

Bhowmik, D., and Zhu, F. (2021). Evasion of intracellular DNA sensing by human herpesviruses. Front. Cell. Infect. Microbiol. 11:647992. doi: 10.3389/fcimb. 2021.647992

Biolatti, M., Dell'Oste, V., Pautasso, S., Gugliesi, F., von Einem, J., Krapp, C., et al. (2018). Human cytomegalovirus tegument protein pp65 (pUL83) dampens type I interferon production by inactivating the DNA sensor cGAS without affecting STING. J. Virol. 92:e01774-17. doi: 10.1128/JVI.01774-17

Birgisdottir, A. B., Lamark, T., and Johansen, T. (2013). The LIR motif - crucial for selective autophagy. J. Cell Sci. 126, 3237-3247. doi: 10.1242/jcs.126128

Bodda, C., Reinert, L. S., Fruhwurth, S., Richardo, T., Sun, C., Zhang, B. C., et al. (2020). HSV1 VP1-2 deubiquitinates STING to block type I interferon expression and promote brain infection. J. Exp. Med. 217:e20191422. doi: $10.1084 /$ jem.20191422

Bouayad, A. (2020). Innate immune evasion by SARS-CoV-2: comparison with SARS-CoV. Rev. Med. Virol. 30, 1-9. doi: 10.1002/rmv.2135

Brezgin, S., Kostyusheva, A., Bayurova, E., Volchkova, E., Gegechkori, V., Gordeychuk, I., et al. (2021). Immunity and viral infections: modulating antiviral response via CRISPR-Cas systems. Viruses 13:1373. doi: 10.3390/ v13071373

Briard, B., Place, D. E., and Kanneganti, T. D. (2020). DNA sensing in the innate immune response. Physiology 35, 112-124. doi: 10.1152/physiol.00022.2019

Carty, M., Guy, C., and Bowie, A. G. (2021). Detection of viral infections by innate immunity. Biochem. Pharmacol. 183:114316. doi: 10.1016/j.bcp.2020.11 4316

Chang, R., Chu, Q., Zheng, W., Zhang, L., and Xu, T. (2020). The Sp1-responsive microRNA-15b negatively regulates Rhabdovirus-triggered innate immune responses in lower vertebrates by targeting TBK1. Front. Immunol. 11:625828. doi: 10.3389/fimmu.2020.625828

Chauveau, L., Bridgeman, A., Tan, T. K., Beveridge, R., Frost, J. N., Rijal, P., et al. (2021). Inclusion of cGAMP within virus-like particle vaccines enhances their immunogenicity. EMBO Rep. 22:e52447. doi: 10.15252/embr.202152447

Chen, Q., Sun, L., and Chen, Z. J. (2016). Regulation and function of the cGASSTING pathway of cytosolic DNA sensing. Nat. Immunol. 17, 1142-1149. doi: 10.1038/ni.3558

Chen, X., Yang, X., Zheng, Y., Yang, Y., Xing, Y., and Chen, Z. (2014). SARS coronavirus papain-like protease inhibits the type I interferon signaling pathway through interaction with the STING-TRAF3-TBK1 complex. Protein Cell 5, 369-381. doi: 10.1007/s13238-014-0026-3

Cheng, Z., Dai, T., He, X., Zhang, Z., Xie, F., Wang, S., et al. (2020). The interactions between cGAS-STING pathway and pathogens. Signal Transduct. Target Ther. $5: 91$

Chiang, C., Liu, G., and Gack, M. U. (2021). Viral evasion of RIG-I-like receptormediated immunity through dysregulation of ubiquitination and ISGylation. Viruses 13:182. doi: 10.3390/v13020182

Chipurupalli, S., Ganesan, R., Dhanabal, S. P., Kumar, M. S., and Robinson, N. (2020). Pharmacological STING activation is a potential alternative to overcome drug-resistance in melanoma. Front. Oncol. 10:758. doi: 10.3389/fonc.2020. 00758

Choi, K. Y., El-Hamdi, N., Hornig, J., and McGregor, A. (2021). Guinea pig cytomegalovirus protective T cell antigen GP83 is a functional pp65 homolog for innate immune evasion and pentamer dependent virus tropism. J. Virol. 95:e00324-21. doi: 10.1128/JVI.00324-21
Cui, Y., Yu, H., Zheng, X., Peng, R., Wang, Q., Zhou, Y., et al. (2017). SENP7 potentiates cGAS activation by relieving SUMO-mediated inhibition of cytosolic DNA sensing. PLoS Pathog. 13:e1006156. doi: 10.1371/journal.ppat. 1006156

Dai, J., Huang, Y. J., He, X., Zhao, M., Wang, X., Liu, Z. S., et al. (2019). Acetylation blocks cGAS activity and inhibits self-DNA-induced autoimmunity. Cell 176, 1447-1460.e14. doi: 10.1016/j.cell.2019.01.016

Davis, M. E., and Gack, M. U. (2015). Ubiquitination in the antiviral immune response. Virology 479-480, 52-65. doi: 10.1016/j.virol.2015.02.033

Duan, Y., Zeng, J., Fan, S., Liao, Y., Feng, M., Wang, L., et al. (2019). Herpes simplex virus type 1-encoded miR-H2-3p manipulates cytosolic DNAstimulated antiviral innate immune response by targeting DDX41. Viruses 11:756. doi: 10.3390/v11080756

Dzimianski, J. V., Scholte, F. E. M., Bergeron, E., and Pegan, S. D. (2019). ISG15: it's complicated. J. Mol. Biol. 431, 4203-4216. doi: 10.1016/j.jmb.2019.03.013

Eaglesham, J. B., and Kranzusch, P. J. (2020). Conserved strategies for pathogen evasion of cGAS-STING immunity. Curr. Opin. Immunol. 66, 27-34. doi: 10.1016/j.coi.2020.04.002

Eaglesham, J. B., McCarty, K. L., and Kranzusch, P. J. (2020). Structures of diverse poxin cGAMP nucleases reveal a widespread role for cGAS-STING evasion in host-pathogen conflict. Elife 9:e59753. doi: 10.7554/eLife.59753

Eaglesham, J. B., Pan, Y., Kupper, T. S., and Kranzusch, P. J. (2019). Viral and metazoan poxins are cGAMP-specific nucleases that restrict cGAS-STING signalling. Nature 566, 259-263.

Fabits, M., Goncalves Magalhaes, V., Chan, B., Girault, V., Elbasani, E., Rossetti, E., et al. (2020). The cytomegalovirus tegument protein UL35 antagonizes pattern recognition receptor-mediated type I IFN transcription. Microorganisms 8:790. doi: 10.3390/microorganisms 8060790

Fang, P., Fang, L., Zhang, H., Xia, S., and Xiao, S. (2021). Functions of coronavirus accessory proteins: overview of the state of the art. Viruses 13:1139. doi: 10. 3390/v13061139

Fang, R., Jiang, Q., Guan, Y., Gao, P., Zhang, R., Zhao, Z., et al. (2021). Golgi apparatus-synthesized sulfated glycosaminoglycans mediate polymerization and activation of the cGAMP sensor STING. Immunity 54, 962-975.e8. doi: 10.1016/j.immuni.2021.03.011

Fang, Y., and Peng, K. (2021). Regulation of innate immune responses by cell death-associated caspases during virus infection. FEBS J.

Feng, L., Li, W., Wu, X., Li, X., Yang, X., Ran, Y., et al. (2021). Human cytomegalovirus UL23 attenuates signal transducer and activator of transcription 1 phosphorylation and type $\mathrm{i}$ interferon response. Front. Microbiol. 12:692515. doi: 10.3389/fmicb.2021.692515

Fischer, T. D., Wang, C., Padman, B. S., Lazarou, M., and Youle, R. J. (2020). STING induces LC3B lipidation onto single-membrane vesicles via the V-ATPase and ATG16L1-WD40 domain. J. Cell Biol. 219:e202009128. doi: 10.1083/jcb. 202009128

Fu, Y. Z., Su, S., Gao, Y. Q., Wang, P. P., Huang, Z. F., Hu, M. M., et al. (2017). Human cytomegalovirus tegument protein UL82 inhibits STING-mediated signaling to evade antiviral immunity. Cell Host Microbe 21, 231-243. doi: 10.1016/j.chom.2017.01.001

Gao, L., Li, K., Zhang, Y., Liu, Y., Liu, C., Zhang, Y., et al. (2019). Inhibition of DNA-sensing pathway by Marek's disease virus VP23 protein through suppression of interferon regulatory factor 7 activation. J. Virol. 93:e01934-18. doi: 10.1128/JVI.01934-18

Ge, Y., Ling, T., Wang, Y., Jia, X., Xie, X., Chen, R., et al. (2021). Degradation of WTAP blocks antiviral responses by reducing the $\mathrm{m}(6)$ A levels of IRF3 and IFNAR1 mRNA. EMBO Rep. 22:e52101. doi: 10.15252/embr.2020 52101

Geddes, V. E. V., de Oliveira, A. S., Tanuri, A., Arruda, E., Ribeiro-Alves, M., and Aguiar, R. S. (2018). MicroRNA and cellular targets profiling reveal miR-217 and miR-576-3p as proviral factors during Oropouche infection. PLoS Negl. Trop. Dis. 12:e0006508. doi: 10.1371/journal.pntd.0006508

Gokhale, N. S., Smith, J. R., Van Gelder, R. D., and Savan, R. (2021). RNA regulatory mechanisms that control antiviral innate immunity. Immunol. Rev.

Gui, X., Yang, H., Li, T., Tan, X., Shi, P., Li, M., et al. (2019). Autophagy induction via STING trafficking is a primordial function of the cGAS pathway. Nature 567, 262-266. doi: 10.1038/s41586-019-1006-9

Guo, Y. K., Ming, S. L., Zeng, L., Chang, W. R., Pan, J. J., Zhang, C., et al. (2021). Inhibition of histone deacetylase 1 suppresses pseudorabies virus infection 
through cGAS-STING antiviral innate immunity. Mol. Immunol. 136, 55-64. doi: 10.1016/j.molimm.2021.05.012

Guo, Y., Jiang, F., Kong, L., Wu, H., Zhang, H., Chen, X., et al. (2021). OTUD5 promotes innate antiviral and antitumor immunity through deubiquitinating and stabilizing STING. Cell Mol. Immunol. 18, 1945-1955. doi: 10.1038/ s41423-020-00531-5

Guo, Y., Li, R., Tan, Z., Shi, J., Fu, Y., Song, Y., et al. (2020). E3 ubiquitin ligase ASB8 negatively regulates interferon via regulating TBK1/IKKi homeostasis. Mol. Immunol. 121, 195-203. doi: 10.1016/j.molimm.2020.03.011

Han, L., Zhuang, M. W., Deng, J., Zheng, Y., Zhang, J., Nan, M. L., et al. (2021). SARS-CoV-2 ORF9b antagonizes type I and III interferons by targeting multiple components of the RIG-I/MDA-5-MAVS, TLR3-TRIF, and CGASSTING signaling pathways. J. Med. Virol. 93, 5376-5389. doi: 10.1002/jmv. 27050

Hilgenfeld, R. (2014). From SARS to MERS: crystallographic studies on coronaviral proteases enable antiviral drug design. FEBS J. 281, 4085-4096. doi: 10.1111/ febs. 12936

Hong, Z., Ma, T., Liu, X., and Wang, C. (2021). cGAS-STING pathway: posttranslational modifications and functions in sterile inflammatory diseases. FEBS J. doi: $10.1111 /$ febs. 16137

Hopfner, K. P., and Hornung, V. (2020). Molecular mechanisms and cellular functions of cGAS-STING signalling. Nat. Rev. Mol. Cell Biol. 21, 501-521.

Hou, P., Lin, Y., Li, Z., Lu, R., Wang, Y., Tian, T., et al. (2021). Autophagy receptor CCDC50 tunes the STING-mediated interferon response in viral infections and autoimmune diseases. Cell Mol. Immunol. 18, 2358-2371. doi: 10.1038/s41423021-00758-w

Hu, X., Tian, J., Kang, H., Guo, D., Liu, J., Liu, D., et al. (2017). Transmissible gastroenteritis virus papain-like protease 1 antagonizes production of interferon-beta through its deubiquitinase activity. Biomed. Res. Int. 2017:7089091. doi: 10.1155/2017/7089091

Huai, W., Liu, X., Wang, C., Zhang, Y., Chen, X., Chen, X., et al. (2019). KAT8 selectively inhibits antiviral immunity by acetylating IRF3. J. Exp. Med. 216, 772-785. doi: 10.1084/jem.20181773

Huang, J., You, H., Su, C., Li, Y., Chen, S., and Zheng, C. (2018). Herpes simplex virus 1 tegument protein VP22 abrogates cGAS/STING-mediated antiviral innate immunity. J. Virol. 92:e00841-18. doi: 10.1128/JVI.00841-18

Huang, L., Liu, H., Zhang, K., Meng, Q., Hu, L., Zhang, Y., et al. (2020). Ubiquitin-conjugating enzyme $2 S$ enhances viral replication by inhibiting type I IFN production through recruiting USP15 to deubiquitinate TBK1. Cell Rep. 32:108044. doi: 10.1016/j.celrep.2020.108044

Kesheh, M. M., Mahmoudvand, S., and Shokri, S. (2021). Long noncoding RNAs in respiratory viruses: a review. Rev. Med. Virol. e2275.

Khan, H., Sumner, R. P., Rasaiyaah, J., Tan, C. P., Rodriguez-Plata, M. T., Van Tulleken, C., et al. (2020). HIV-1 Vpr antagonizes innate immune activation by targeting karyopherin-mediated NF-kappaB/IRF3 nuclear transport. Elife 9:e60821. doi: 10.7554/eLife.60821

Kikkert, M. (2020). Innate immune evasion by human respiratory RNA viruses. J. Innate Immun. 12, 4-20.

Klemm, T., Ebert, G., Calleja, D. J., Allison, C. C., Richardson, L. W., Bernardini, J. P., et al. (2020). Mechanism and inhibition of the papain-like protease, PLpro, of SARS-CoV-2. EMBO J. 39:e106275.

Kumari, P., and Kumar, H. (2018). Viral deubiquitinases: role in evasion of anti-viral innate immunity. Crit. Rev. Microbiol. 44, 304-317.

Kumari, P., Saha, I., Narayanan, A., Narayanan, S., Takaoka, A., Kumar, N. S., et al. (2017). Essential role of HCMV deubiquitinase in promoting oncogenesis by targeting anti-viral innate immune signaling pathways. Cell Death Dis. 8:e3078. doi: 10.1038/cddis.2017.461

Lange, P. T., White, M. C., and Damania, B. (2021). Activation and evasion of innate immunity by gammaherpesviruses. J. Mol. Biol. 167214. doi: 10.1016/ j.jmb.2021.167214

Li, C., Zhang, L., Qian, D., Cheng, M., Hu, H., Hong, Z., et al. (2021b). RNF111facilitated neddylation potentiates cGAS-mediated antiviral innate immune response. PLoS Pathog. 17:e1009401. doi: 10.1371/journal.ppat.1009401

Li, C., Feng, L., Luo, W. W., Lei, C. Q., Li, M., and Shu, H. B. (2021a). The RNAbinding protein LUC7L2 mediates MITA/STING intron retention to negatively regulate innate antiviral response. Cell Discov. 7:46. doi: 10.1038/s41421-02100277-y
Li, K., Liu, Y., Xu, Z., Zhang, Y., Luo, D., Gao, Y., et al. (2019). Avian oncogenic herpesvirus antagonizes the cGAS-STING DNA-sensing pathway to mediate immune evasion. PLoS Pathog. 15:e1007999. doi: 10.1371/journal.ppat.1007999

Li, P., and Chang, M. (2021). Roles of PRR-mediated signaling pathways in the regulation of oxidative stress and inflammatory diseases. Int. J. Mol. Sci. 22:7688. doi: 10.3390/ijms22147688

Li, Q., Lin, L., Tong, Y., Liu, Y., Mou, J., Wang, X., et al. (2018). TRIM29 negatively controls antiviral immune response through targeting STING for degradation. Cell Discov. 4:13.

Li, Z., Cai, S., Sun, Y., Li, L., Ding, S., and Wang, X. (2020). When STING meets viruses: sensing, trafficking and response. Front. Immunol. 11:2064. doi: 10. 3389/fimmu.2020.02064

Liang, Q., Seo, G. J., Choi, Y. J., Kwak, M. J., Ge, J., Rodgers, M. A., et al. (2014). Crosstalk between the cGAS DNA sensor and Beclin-1 autophagy protein shapes innate antimicrobial immune responses. Cell Host Microbe 15, 228-238. doi: 10.1016/j.chom.2014.01.009

Lin, M., Zhao, Z., Yang, Z., Meng, Q., Tan, P., Xie, W., et al. (2016). USP38 inhibits type I interferon signaling by editing TBK1 ubiquitination through NLRP4 signalosome. Mol. Cell 64, 267-281. doi: 10.1016/j.molcel.2016.08.029

Lin, Y., and Zheng, C. (2019). A tug of war: DNA-sensing antiviral innate immunity and herpes simplex virus type I infection. Front. Microbiol. 10:2627. doi: 10. 3389/fmicb.2019.02627

Liu, D., Wu, H., Wang, C., Li, Y., Tian, H., Siraj, S., et al. (2019). STING directly activates autophagy to tune the innate immune response. Cell Death Differ. 26, 1735-1749. doi: 10.1038/s41418-018-0251-z

Liu, S., Chen, S., Li, X., Wu, S., Zhang, Q., Jin, Q., et al. (2017). Lck/Hck/Fgrmediated tyrosine phosphorylation negatively regulates TBK1 to restrain innate antiviral responses. Cell Host Microbe 21, 754-768.e5. doi: 10.1016/j.chom. 2017.05.010

Liu, W., Reyes, H. M., Yang, J. F., Li, Y., Stewart, K. M., Basil, M. C., et al. (2021). Activation of STING signaling pathway effectively blocks human coronavirus infection. J. Virol. 95:e00490-21.

Liu, X., Main, D., Ma, Y., and He, B. (2018). Herpes simplex virus 1 inhibits TANKbinding kinase 1 through formation of the Us11-Hsp90 complex. J. Virol. 92:e00402-18. doi: 10.1128/JVI.00402-18

Ma, D., Yang, M., Wang, Q., Sun, C., Shi, H., Jing, W., et al. (2021). Arginine methyltransferase PRMT5 negatively regulates cGAS-mediated antiviral immune response. Sci. Adv. 7:eabc1834. doi: 10.1126/sciadv.abc1834

Mathieu, N. A., Paparisto, E., Barr, S. D., and Spratt, D. E. (2021). HERC5 and the ISGylation pathway: critical modulators of the antiviral immune response. Viruses 13:1102. doi: 10.3390/v13061102

Morchikh, M., Cribier, A., Raffel, R., Amraoui, S., Cau, J., Severac, D., et al. (2017). HEXIM1 and NEAT1 long non-coding RNA form a multi-subunit complex that regulates DNA-mediated innate immune response. Mol. Cell 67, 387-399.e5. doi: 10.1016/j.molcel.2017.06.020

Morehouse, B. R., Govande, A. A., Millman, A., Keszei, A. F. A., Lowey, B., Ofir, G., et al. (2020). STING cyclic dinucleotide sensing originated in bacteria. Nature 586, 429-433. doi: 10.1038/s41586-020-2719-5

Motwani, M., Pesiridis, S., and Fitzgerald, K. A. (2019). DNA sensing by the cGAS-STING pathway in health and disease. Nat. Rev. Genet. 20, 657-674. doi: 10.1038/s41576-019-0151-1

Nanbo, A., Furuyama, W., and Lin, Z. (2021). RNA virus-encoded miRNAs: current insights and future challenges. Front. Microbiol. 12:679210. doi: 10. $3389 /$ fmicb. 2021.679210

Phelan, T., Little, M. A., and Brady, G. (2020). Targeting of the cGAS-STING system by DNA viruses. Biochem. Pharmacol. 174:113831. doi: 10.1016/j.bcp. 2020.113831

Proulx, J., Borgmann, K., and Park, I. W. (2021). Role of virally-encoded deubiquitinating enzymes in regulation of the virus life cycle. Int. J. Mol. Sci. 22:4438. doi: 10.3390/ijms22094438

Qu, Z., Meng, F., Shi, J., Deng, G., Zeng, X., Ge, J., et al. (2021). A novel intronic circular RNA antagonizes influenza virus by absorbing a microRNA that degrades CREBBP and accelerating IFN-beta production. mBio 12:e0101721. doi: 10.1128/mBio.01017-21

Rui, Y., Su, J., Shen, S., Hu, Y., Huang, D., Zheng, W., et al. (2021). Unique and complementary suppression of cGAS-STING and RNA sensing- triggered innate immune responses by SARS-CoV-2 proteins. Signal Transduct. Target Ther. 6:123. doi: 10.1038/s41392-021-00515-5 
Sausen, D. G., Reed, K. M., Bhutta, M. S., Gallo, E. S., and Borenstein, R. (2021). Evasion of the host immune response by betaherpesviruses. Int. J. Mol. Sci. 22:7503. doi: $10.3390 /$ ijms 22147503

Schneider, W. M., Chevillotte, M. D., and Rice, C. M. (2014). Interferon-stimulated genes: a complex web of host defenses. Annu. Rev. Immunol. 32, 513-545. doi: 10.1146/annurev-immunol-032713-120231

Schwanke, H., Stempel, M., and Brinkmann, M. M. (2020). Of keeping and tipping the balance: host regulation and viral modulation of IRF3-dependent IFNB1 expression. Viruses 12:733. doi: 10.3390/v12070733

Shang, G., Zhang, C., Chen, Z. J., Bai, X. C., and Zhang, X. (2019). Cryo-EM structures of STING reveal its mechanism of activation by cyclic GMP-AMP. Nature 567, 389-393. doi: 10.1038/s41586-019-0998-5

Shi, H. X., Yang, K., Liu, X., Liu, X. Y., Wei, B., Shan, Y. F., et al. (2010). Positive regulation of interferon regulatory factor 3 activation by Herc5 via ISG15 modification. Mol. Cell Biol. 30, 2424-2436. doi: 10.1128/MCB.01466-09

Shin, D., Mukherjee, R., Grewe, D., Bojkova, D., Baek, K., Bhattacharya, A., et al. (2020). Papain-like protease regulates SARS-CoV-2 viral spread and innate immunity. Nature 587, 657-662. doi: 10.1038/s41586-020-2601-5

Song, B., Liu, D., Greco, T. M., and Cristea, I. M. (2021). Post-translational modification control of viral DNA sensors and innate immune signaling. Adv. Virus Res. 109, 163-199. doi: 10.1016/bs.aivir.2021.03.001

Song, G., Liu, B., Li, Z., Wu, H., Wang, P., Zhao, K., et al. (2016). E3 ubiquitin ligase RNF128 promotes innate antiviral immunity through K63linked ubiquitination of TBK1. Nat. Immunol. 17, 1342-1351. doi: 10.1038/ ni. 3588

Song, Z. M., Lin, H., Yi, X. M., Guo, W., Hu, M. M., and Shu, H. B. (2020). KAT5 acetylates cGAS to promote innate immune response to DNA virus. Proc. Natl. Acad. Sci. U.S.A. 117, 21568-21575. doi: 10.1073/pnas.1922330117

Sparrer, K. M. J., Gableske, S., Zurenski, M. A., Parker, Z. M., Full, F., Baumgart, G. J., et al. (2017). TRIM23 mediates virus-induced autophagy via activation of TBK1. Nat. Microbiol. 2, 1543-1557. doi: 10.1038/s41564-017-0017-2

Su, C. I., Kao, Y. T., Chang, C. C., Chang, Y., Ho, T. S., Sun, H. S., et al. (2020). DNA-induced 2'3'-cGAMP enhances haplotype-specific human STING cleavage by dengue protease. Proc. Natl. Acad. Sci. U.S.A. 117, 15947-15954. doi: $10.1073 /$ pnas.1922243117

Su, C., and Zheng, C. (2017). Herpes simplex virus 1 abrogates the cGAS/STINGmediated cytosolic DNA-sensing pathway via its virion host shutoff protein, UL41. J. Virol. 91:e02414-16. doi: 10.1128/JVI.02414-16

Su, J., Rui, Y., Lou, M., Yin, L., Xiong, H., Zhou, Z., et al. (2019). HIV-2/SIV Vpx targets a novel functional domain of STING to selectively inhibit cGASSTING-mediated NF-kappaB signalling. Nat. Microbiol. 4, 2552-2564. doi: 10.1038/s41564-019-0585-4

Sun, X., Liu, T., Zhao, J., Xia, H., Xie, J., Guo, Y., et al. (2020). DNA-PK deficiency potentiates cGAS-mediated antiviral innate immunity. Nat. Commun. 11:6182. doi: 10.1038/s41467-020-19941-0

Tang, J. L., Yang, Q., Xu, C. H., Zhao, H., Liu, Y. L., Liu, C. Y., et al. (2021). Histone deacetylase 3 promotes innate antiviral immunity through deacetylation of TBK1. Protein Cell 12, 261-278. doi: 10.1007/s13238-020-00751-5

Tian, M., Liu, W., Zhang, Q., Huang, Y., Li, W., Wang, W., et al. (2020). MYSM1 represses innate immunity and autoimmunity through suppressing the cGASSTING pathway. Cell Rep. 33:108297. doi: 10.1016/j.celrep.2020.108297

Uhlorn, B. L., Jackson, R., Li, S., Bratton, S. M., Van Doorslaer, K., and Campos, S. K. (2020). Vesicular trafficking permits evasion of cGAS/STING surveillance during initial human papillomavirus infection. PLoS Pathog. 16:e1009028. doi: 10.1371/journal.ppat.1009028

Wan, D., Jiang, W., and Hao, J. (2020). Research advances in how the cGAS-STING pathway controls the cellular inflammatory response. Front. Immunol. 11:615. doi: 10.3389/fimmu.2020.00615

Wang, C., Wang, Q., Xu, X., Xie, B., Zhao, Y., Li, N., et al. (2017). The methyltransferase NSD3 promotes antiviral innate immunity via direct lysine methylation of IRF3. J. Exp. Med. 214, 3597-3610. doi: 10.1084/jem.20170856

Wang, X., Wu, J., Wu, Y., Chen, H., Zhang, S., Li, J., et al. (2018). Inhibition of cGAS-STING-TBK1 signaling pathway by DP96R of ASFV China 2018/1. Biochem. Biophys. Res. Commun. 506, 437-443. doi: 10.1016/j.bbrc.2018.10.103

Wang, Y., Luo, J., Alu, A., Han, X., Wei, Y., and Wei, X. (2020a). cGAS-STING pathway in cancer biotherapy. Mol. Cancer 19:136.

Wang, Y., Wang, P., Zhang, Y., Xu, J., Li, Z., Li, Z., et al. (2020b). Decreased expression of the host long-noncoding RNA-GM facilitates viral escape by inhibiting the kinase activity TBK1 via S-glutathionylation. Immunity 53, 11681181.e7. doi: 10.1016/j.immuni.2020.11.010

Wu, J. J., Zhao, L., Han, B. B., Hu, H. G., Zhang, B. D., Li, W. H., et al. (2021). A novel STING agonist for cancer immunotherapy and a SARS-CoV-2 vaccine adjuvant. Chem. Commun. 57, 504-507. doi: 10.1039/d0cc06959k

$\mathrm{Wu}, \mathrm{Y}$., and Li, S. (2020). Role of post-translational modifications of cGAS in innate immunity. Int. J. Mol. Sci. 21:7842. doi: 10.3390/ijms21217842

Xia, P., Ye, B., Wang, S., Zhu, X., Du, Y., Xiong, Z., et al. (2016). Glutamylation of the DNA sensor cGAS regulates its binding and synthase activity in antiviral immunity. Nat. Immunol. 17, 369-378. doi: 10.1038/ni.3356

Xiao, Q., McAtee, C. K., and Su, X. (2021). Phase separation in immune signalling. Nat. Rev. Immunol.

Xie, W., Jin, S., Zhang, C., Yang, S., Wu, Y., Zhao, Y., et al. (2021). Selective autophagy controls the stability of TBK1 via NEDD4 to balance host defense. Cell Death Differ. doi: 10.1038/s41418-021-00833-9

Xu, G., Liu, C., Zhou, S., Li, Q., Feng, Y., Sun, P., et al. (2021). Viral tegument proteins restrict cGAS-DNA phase separation to mediate immune evasion. Mol. Cell 81, 2823-2837.e9. doi: 10.1016/j.molcel.2021.05.002

$\mathrm{Xu}, \mathrm{T}$., Chu, Q., and Cui, J. (2018). Rhabdovirus-inducible microRNA-210 modulates antiviral innate immune response via targeting STING/MITA in fish. J. Immunol. 201, 982-994. doi: 10.4049/jimmunol.1800377

Xu, Y., Chen, K., Pan, J., Lei, Y., Zhang, D., Fang, L., et al. (2021). Repurposing clinically approved drugs for COVID-19 treatment targeting SARS-CoV-2 papain-like protease. Int. J. Biol. Macromol. 188, 137-146. doi: 10.1016/j. ijbiomac.2021.07.184

Yamashiro, L. H., Wilson, S. C., Morrison, H. M., Karalis, V., Chung, J.-Y. J., Chen, K. J., et al. (2019). STING controls Herpes Simplex Virus in vivo independent of type I interferon induction. bioRxiv [Preprint]. doi: 10.1101/ 2019.12.12.874792.c

Yang, B., Liu, Y., Cui, Y., Song, D., Zhang, G., Ma, S., et al. (2020). RNF90 negatively regulates cellular antiviral responses by targeting MITA for degradation. PLoS Pathog. 16:e1008387. doi: 10.1371/journal.ppat.1008387

Yang, L., Wang, M., Cheng, A., Yang, Q., Wu, Y., Jia, R., et al. (2019). Innate immune evasion of alphaherpesvirus tegument proteins. Front. Immunol. 10:2196. doi: 10.3389/fimmu.2019.02196

Ye, L., Zhang, Q., Liuyu, T., Xu, Z., Zhang, M. X., Luo, M. H., et al. (2019). USP49 negatively regulates cellular antiviral responses via deconjugating K63-linked ubiquitination of MITA. PLoS Pathog. 15:e1007680. doi: 10.1371/journal.ppat. 1007680

Ye, R., Su, C., Xu, H., and Zheng, C. (2017). Herpes simplex virus 1 ubiquitinspecific protease UL36 abrogates NF-kappaB activation in DNA sensing signal pathway. J. Virol. 91:e02417-16. doi: 10.1128/JVI.02417-16

You, H., Zheng, S., Huang, Z., Lin, Y., Shen, Q., and Zheng, C. (2019). Herpes simplex virus 1 tegument protein UL46 inhibits TANK-binding kinase 1mediated signaling. mBio 10:e00919-19. doi: 10.1128/mBio.00919-19

Yu, H., Bruneau, R. C., Brennan, G., and Rothenburg, S. (2021). Battle royale: innate recognition of poxviruses and viral immune evasion. Biomedicines 9:765. doi: 10.3390/biomedicines 9070765

Yu, P., Miao, Z., Li, Y., Bansal, R., Peppelenbosch, M. P., and Pan, Q. (2021). cGAS-STING effectively restricts murine norovirus infection but antagonizes the antiviral action of N-terminus of RIG-I in mouse macrophages. Gut Microbes 13:1959839. doi: 10.1080/19490976.2021.195 9839

Yum, S., Li, M., Fang, Y., and Chen, Z. J. (2021). TBK1 recruitment to STING activates both IRF3 and NF-kappaB that mediate immune defense against tumors and viral infections. Proc. Natl. Acad. Sci. U.S.A. 118:e2100225118. doi: $10.1073 /$ pnas.2100225118

Zhang, D., Su, C., and Zheng, C. (2016). Herpes simplex virus 1 serine protease VP24 blocks the DNA-sensing signal pathway by abrogating activation of interferon regulatory factor 3. J. Virol. 90, 5824-5829. doi: 10.1128/JVI.00 186-16

Zhang, J., Hu, M. M., Wang, Y. Y., and Shu, H. B. (2012). TRIM32 protein modulates type I interferon induction and cellular antiviral response by targeting MITA/STING protein for K63-linked ubiquitination. J. Biol. Chem. 287, 28646-28655. doi: 10.1074/jbc.M112.362608

Zhang, J., Li, Z., Huang, J., Yin, H., Tian, J., and Qu, L. (2019). miR-26a inhibits feline herpesvirus 1 replication by targeting SOCS5 and promoting type I interferon signaling. Viruses 12:2. doi: 10.3390/v12010002 
Zhang, J., Zhao, J., Xu, S., Li, J., He, S., Zeng, Y., et al. (2018). Species-specific deamidation of cGAS by herpes simplex virus UL37 protein facilitates viral replication. Cell Host Microbe 24, 234-248.e5. doi: 10.1016/j.chom.2018.07.004

Zhang, Q., Song, X., Ma, P., Lv, L., Zhang, Y., Deng, J., et al. (2021). Human cytomegalovirus miR-US33as-5p targets IFNAR1 to achieve immune evasion during both lytic and latent infection. Front. Immunol. 12:628364. doi: 10.3389/ fimmu.2021.628364

Zhang, R., Qin, X., Yang, Y., Zhu, X., Zhao, S., Zhang, Z., et al. (2021). STING1 is essential for an RNA-virus triggered autophagy. Autophagy 1-13. doi: 10.1080/ 15548627.2021.1959086

Zhang, X., Bai, X. C., and Chen, Z. J. (2020c). Structures and mechanisms in the cGAS-STING innate immunity pathway. Immunity 53, 43-53. doi: 10.1016/j. immuni.2020.05.013

Zhang, H. Y., Liao, B. W., Xu, Z. S., Ran, Y., Wang, D. P., Yang, Y., et al. (2020a). USP44 positively regulates innate immune response to DNA viruses through deubiquitinating MITA. PLoS Pathog. 16:e1008178. doi: 10.1371/journal.ppat. 1008178

Zhang, Z., Wang, D., Wang, P., Zhao, Y., and You, F. (2020e). OTUD1 negatively regulates type I IFN induction by disrupting noncanonical ubiquitination of IRF3. J. Immunol. 204, 1904-1918. doi: 10.4049/jimmunol.1900305

Zhang, J., Li, Z., Huang, J., Chen, S., Yin, H., Tian, J., et al. (2020b). miR-101 inhibits feline herpesvirus 1 replication by targeting cellular suppressor of cytokine signaling 5 (SOCS5). Vet. Microbiol. 245:108707. doi: 10.1016/j.vetmic.2020. 108707

Zhang, Y., Li, M., Li, L., Qian, G., Wang, Y., Chen, Z., et al. (2020d). Beta-arrestin 2 as an activator of cGAS-STING signaling and target of viral immune evasion. Nat. Commun. 11:6000. doi: 10.1038/s41467-020-19849-9

Zhao, B., Du, F., Xu, P., Shu, C., Sankaran, B., Bell, S. L., et al. (2019). A conserved PLPLRT/SD motif of STING mediates the recruitment and activation of TBK1. Nature 569, 718-722. doi: 10.1038/s41586-019-1228-x

Zhao, H., Wu, L., Yan, G., Chen, Y., Zhou, M., Wu, Y., et al. (2021). Inflammation and tumor progression: signaling pathways and targeted intervention. Signal Transduct. Target Ther. 6:263.
Zheng, C. (2018). Evasion of cytosolic DNA-stimulated innate immune responses by herpes simplex virus 1. J. Virol. 92:e0099-17.

Zheng, C. (2021). The emerging roles of NOD-like receptors in antiviral innate immune signaling pathways. Int. J. Biol. Macromol. 169, 407-413. doi: 10.1016/ j.ijbiomac.2020.12.127

Zhu, H., and Zheng, C. (2020). The race between host antiviral innate immunity and the immune evasion strategies of herpes simplex virus 1. Microbiol. Mol. Biol. Rev. 84:e0099-20. doi: 10.1128/MMBR.00 099-20

Zhu, Q., Zhang, Y., Wang, L., Yao, X., Wu, D., Cheng, J., et al. (2021). Inhibition of coronavirus infection by a synthetic STING agonist in primary human airway system. Antiviral Res. 187:105015. doi: 10.1016/j.antiviral.2021.10 5015

Zou, H. M., Huang, Z. F., Yang, Y., Luo, W. W., Wang, S. Y., Luo, M. H., et al. (2020). Human cytomegalovirus protein UL94 targets MITA to evade the antiviral immune response. J. Virol. 94:e00022-20. doi: 10.1128/JVI.00022-20

Conflict of Interest: The authors declare that the research was conducted in the absence of any commercial or financial relationships that could be construed as a potential conflict of interest.

Publisher's Note: All claims expressed in this article are solely those of the authors and do not necessarily represent those of their affiliated organizations, or those of the publisher, the editors and the reviewers. Any product that may be evaluated in this article, or claim that may be made by its manufacturer, is not guaranteed or endorsed by the publisher.

Copyright (c) $2021 \mathrm{Hu}$, Pan, Yin, Wang, Cui and Wang. This is an open-access article distributed under the terms of the Creative Commons Attribution License (CC BY). The use, distribution or reproduction in other forums is permitted, provided the original author(s) and the copyright owner(s) are credited and that the original publication in this journal is cited, in accordance with accepted academic practice. No use, distribution or reproduction is permitted which does not comply with these terms. 